\title{
Option Portfolio Management in a Risk-Neutral World
}

\author{
Dmitry Jurievich Golembiovsky ${ }^{1,2 *}$, Anatoly Markovich Abramov ${ }^{3}$ \\ ${ }^{1}$ Moscow State University, Moscow, Russia \\ ${ }^{2}$ Synergy University, Moscow, Russia \\ ${ }^{3}$ International Monetary Fund, Washington DC, USA \\ Email: ^golemb@cs.msu.su
}

How to cite this paper: Golembiovsky, D.J. and Abramov, A.M. (2018) Option Portfolio Management in a Risk-Neutral World. Journal of Mathematical Finance, 8 , 710-733.

https://doi.org/10.4236/jmf.2018.84044

Received: September 18, 2018

Accepted: November 25, 2018

Published: November 28, 2018

Copyright (c) 2018 by authors and Scientific Research Publishing Inc. This work is licensed under the Creative Commons Attribution International License (CC BY 4.0).

http://creativecommons.org/licenses/by/4.0/

\begin{abstract}
The most commonly used strategy of the speculative investments in options is a statistical arbitrage between the objective underlying price distribution which the price is following and the risk-neutral distribution on the basis of which options were priced. This article investigates an alternative approach which does not demand these two distributions to be different. Instead, it uses a periodical roll-over of an investment horizon with including options of the next expirations in the portfolio. We consider a risk-neutral world where the real distribution coincides with the risk-neutral distribution as a model of the market. In such a market, the expected return from investments in any option portfolio corresponds with the risk-free rate. However, it is possible to construct and manage the portfolio dynamically in such a way that it provides higher return with a probability close to unity or lower return (possibly a large negative return) with a given very low probability. To optimize the portfolio a stochastic program with the approximative safety-first criterion for option portfolio was developed along with the corresponding multinomial scenario tree. The results of the Monte-Carlo simulation of the portfolio management are presented. The very low probability of loss during option portfolio management is provided by the strategy with periodical rolling horizon of the optimization. The developed portfolio management strategy can be used as a basis for constructing trading strategies for the real option markets.
\end{abstract}

\section{Keywords}

Risk-Neutral World, Option Portfolio, Standard Portfolio Analysis of Risk, Stochastic Programming, Safety-First Criterion, Scenario Tree, Monte-Carlo Simulation 


\section{Introduction}

Let's consider a market consisting of a risky and a risk-free asset. Such a market is called complete if some self-financing (without external capital inflow) portfolio of these two assets can replicate any contingent claim. It means the following: there exists a strategy of the portfolio management such that the terminal value of the portfolio equals the value of the contingent claim at expire with the probability of 1 .

It is said that the market admits arbitrage, if it is possible to construct and manage a self-financing portfolio of these two assets which satisfies the following conditions: its initial value is zero; its terminal value is non-negative with the probability of 1 and greater than zero with some positive probability. The market which does not admit arbitrage is arbitrage-free.

The so-called first fundamental theorem of asset pricing (for example, see [1]) states that a market is arbitrage-free if and only if for the risky asset there exists an equivalent martingale measure. It is such a probability measure, under which the process of the risky asset price discounted by the risk-free rate is a martingale. At the same time, this measure is equivalent to the objective probability measure which the risky asset is following (it is equaled to zero only if the objective measure equals zero).

According to the second fundamental theorem of asset pricing a market is complete if and only if the equivalent martingale measure is unique. In a complete market an arbitrage-free price of a contingent claim is unique and equals the initial value of the replicating portfolio. On the other hand, it can be proved that the price of a European contingent claim equals its expected value under the martingale probability measure discounted by the risk-free interest rate.

Existence of a martingale measure for a market excludes arbitrage possibilities defined therein. However, if the objective (real) and the equivalent martingale measure are different, there can exist a statistical arbitrage possibility. Actually, if the discounted expected value of a contingent claim under objective probability measure is more than the expected value under the martingale measure it is reasonable to borrow enough money at the risk-free interest rate, to buy the claim and wait till its expiration. In opposite situation, an arbitrager should sell the claim, invest the proceeds of the short sale at the risk-free interest rate and wait the expiration too. In both cases the expected return is positive. However, the loss can be gotten in some cases of course.

Such statistical arbitrage often takes form of volatility trading: investors buy options if the growth of volatility is expected and sell options in the opposite case (for example, see [2]). It should be marked that in a real market the statistical arbitrage is an enough complex problem because the objective distribution is changing permanently and investors do not know it precisely.

The market where the discounted risky asset price moves per the martingale measure is called the risk-neutral world. Such a world can be considered as a model of the real market where the statistical arbitrage in the fore mentioned 
sense is impossible. If we buy a claim in the risk-neutral world, the expected return of the investment corresponds to the risk-free interest rate. If we sell a claim and put the premium on a risk-free account, the expected return from the investment will be zero. Consequently, the expected return equals the risk-free rate for any investment in contingent portfolio in a risk-neutral world. The same yield can be obtained investing in the risk-free asset. Portfolio optimization considering the expected portfolio return must lead to the portfolio which consists of the risk-free asset only if risk aversion is employed by the program.

If we produce a perfect hedge of a contingent portfolio using a portfolio of the underlying and risk-free assets (even in a non-risk-neutral world), the result will be obtained with the probability of 1 . The greater return can be obtained only with some probability that is less than unity in a risk-neutral world. One of the possibilities to do this is the partial hedging which is cheaper than the perfect hedging [3]. This paper develops the optimal self-financing strategy of hedging an option by the underlying asset and the risk-free asset which ensures a desirable pay-off in all discrete scenarios except one which is the most unlikely scenario. This scenario results in a loss which is more if the starting value of the hedging portfolio is less and vice versa. So, it is possible, for example, to sell an option at its theoretical price, keep a part of the premium in the risk-free account and apply the developed hedging strategy using the rest of the money. If the mentioned scenario does not occur, the investor will have a profit without initial input.

This study shows that it is possible to make more than the risk-free rate with the probability close to unity in a risk-neutral world by dynamic managing of option portfolio. To optimize the portfolio we use the multistage stochastic programming with some kind of chance constraints ([4]-[16]).

In our previous paper [17] option portfolio with one expiration date was considered. The fulfilled case study showed that the probability of not reaching the planed profit was quite high: 6 experiments conducted on one month option portfolio management out of 100 finished with a loss. A few contributions have been made to improve the performance now:

- The stochastic program suggested in [17] is generalized for including options of different expirations in the portfolio,

- Heuristics for solving the mix integer problem with binary variables is suggested which reduces the required computer time dramatically,

- A strategy of using the stochastic program with rolling planning horizon is developed.

The paper is organized as follows. Section 2 contains a very simple example of the statistical arbitrage with options using roll-over of the planning horizon. In Section 3 the description of exchange collateral system SPAN is given. An example of the initial margin calculation for a real market is provided. A heuristic for solving safety-first optimization problem is suggested in Section 4. Section 5 describes the scenario tree construction and develops the stochastic optimization problem for option portfolio with an arbitrary number of expirations. It begins 
with formulation of the main assumptions which the optimization program is based on. One of them is collateral requirements based on SPAN system.

Section 6 presents the case studies which were performed by Monte-Carlo simulation of option portfolio management. At first, we compare the simulation of one expiration options portfolio solving the exact mixed-Boolean problem and solving the same problem by application of the suggested heuristic method. Then the results obtained during simulation of the portfolio with rolling planning horizon in a risk-neutral world are provided. This portfolio included options of one or two different expirations. We conclude in Section 8.

\section{An Example of the Statistical Arbitrage Based on Roll-Over}

This section talks about a very simple example of the statistical arbitrage without optimization. It is illustrated in Figure 1. The horizontal axis is used for mapping the underlying price. The vertical axis shows the premiums of options. The diagonal axis shows the time. The trajectory of the underlying asset price is shown in red.

The underlying price is equal to 100 on the day 1 . We short a strangle with the strike prices of 96 and 104 which expires on the last day of the current month. The corresponding options are displayed by their profit functions on the vertical and horizontal axes relevant to the end of the first month. The premium of this Put is 0.80 and the premium of this Call is 0.87 . So, if both the options expire out of the money we will have profit $0.80+0.87=1.67$ at the end of the first month.

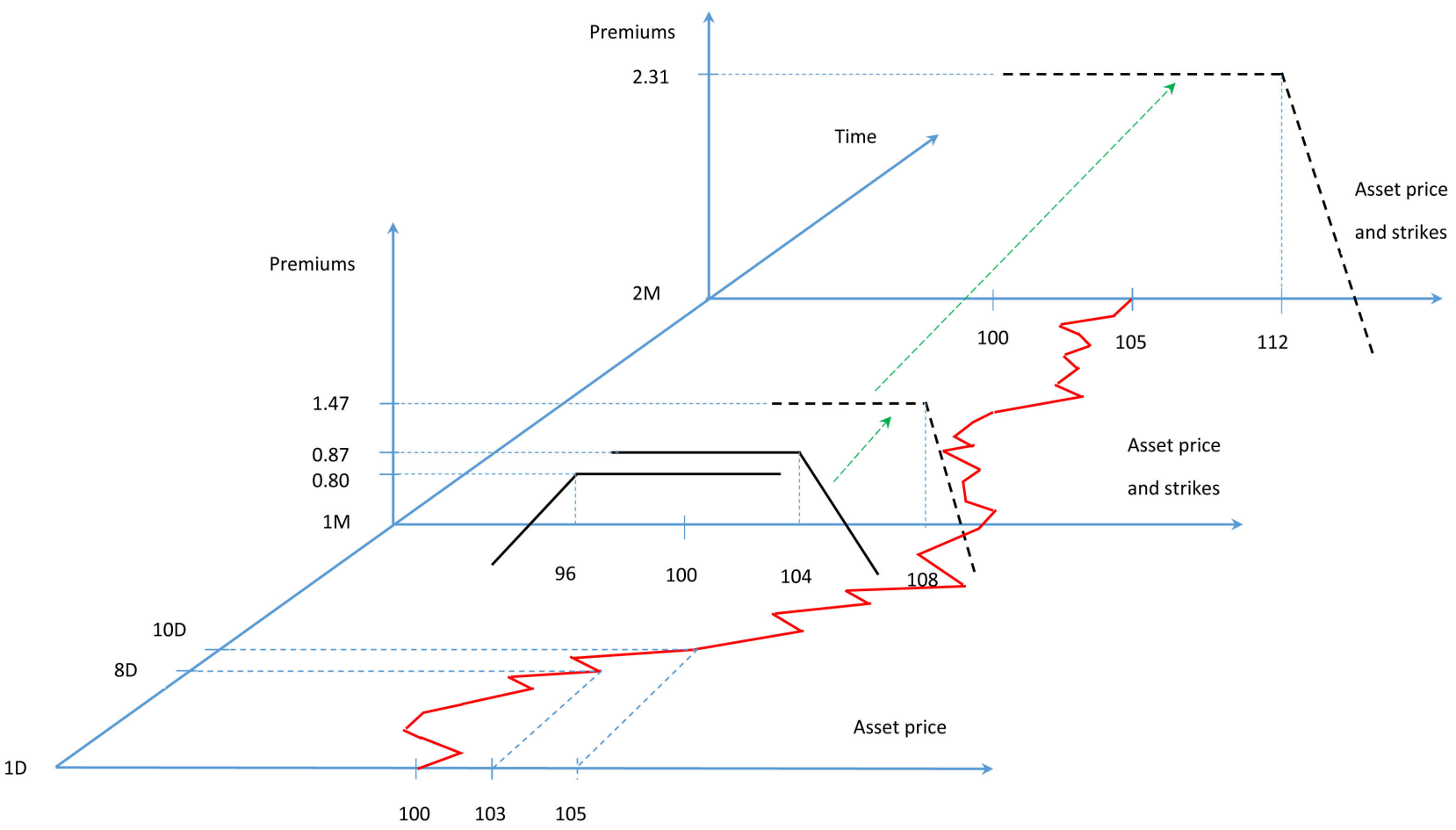

Figure 1. A simple example of the statistical arbitrage. 
The rows of Table 1 indicate the options that the portfolio consists after the corresponding day. The current portfolio is shown by the row of Table 1 which accords with day 1 .

In the example the asset price grows after portfolio creating and our Call option becomes close to the money. On the 8th day of the first month the underlying price is 103. The Put and Call options costs 0.12 and 1.42 respectively. We close our position in Call option and short 4 Call options the same expiration with the strike price of 108 . Each of them costs 0.37 . (This transfer is symbolized by green dotted arrow in Figure 1. The profit function of new options is shown by the dotted line). So, we pay 1.42 and receive $0.37 \times 4=1.47$, therefore the balance is $1.47-1.42=0.05$. Now, if all our options expire out of the money, we will get a profit $1.67+0.05=1.72$. The portfolio content is displayed in the row of Table 1 relevant to the day 8 .

Unfortunately, the underlying price increases again. On the 10th day of the month it reaches 105. At this moment premium of our Put option is 0.02 , each Call options of the strike 108 costs 0.66 .

All exchanges require collateral for any option positions (look [18] for reference). If our cash is limited, there can be a moment, when the better decision is to transfer some of our positions to the next month, but not to a much further strike of the current month. The reason is the following: options of the next expiration are more expensive. At the same time their collateral is usually not far from the collateral of the current month options.

Let us assume that on the 10th day we decide to transfer Call options to the next month. We short Call options of the strike 112. Their price equals 0.77 on the considered day. Suppose our cash balance permits us to sell only three such options not violating the collateral requirements. In this case the proceeds is 0.77 $\times 3=2.31$ for shorting options of the next month. Simultaneously, we pay $0.66 \times$ $4=2.64$ for closing Call option positions of the nearest month. Our balance now is $2.31-2.64=-0.33$. So, if all our options expire out of the money we will receive the profit $1.72-0.33=1.39$.

This transfer is shown by another green dotted arrow in Figure 1. The profit function of the new sold options is displayed by the black dotted line on the vertical and horizontal axes relevant to the end of the second month. The gotten portfolio is given by the last row of Table 1 .

Per Figure 1, the asset price does not essentially increase henceforth. Its final value at the end of the second month is 105 . Both option positions expire out of the money and we lock the considered profit by the end of the second month.

Table 1. Statistical arbitrage portfolio.

\begin{tabular}{|c|c|c|c|c|c|c|c|}
\hline \multirow{2}{*}{ Day } & \multicolumn{3}{|c|}{ Put } & \multicolumn{3}{|c|}{ Call } & \multirow{2}{*}{$\begin{array}{c}\text { Earn if options expire } \\
\text { out of the money }\end{array}$} \\
\hline & Position & Strike & Expiration & Position & Strike & Expiration & \\
\hline 1 & -1 & 96 & $1 \mathrm{M}$ & -1 & 104 & $1 \mathrm{M}$ & 1.67 \\
\hline 8 & -1 & 96 & $1 \mathrm{M}$ & -4 & 108 & $1 \mathrm{M}$ & 1.72 \\
\hline 10 & -1 & 96 & $1 \mathrm{M}$ & -3 & 112 & $2 \mathrm{M}$ & 1.39 \\
\hline
\end{tabular}


This paper is devoted to developing the statistical arbitrage strategy based on the multistage stochastic programming. Using optimization more complex option portfolios, which can include long and short positions, are constructed.

\section{The Scanning Risk of Option Portfolio}

As mentioned, to trade options on a real exchange a collateral is required. It consists of two following terms: net liquidation value of option portfolio and maintenance or initial margin. Net liquidation value is the current portfolio value with an inverse sign. So, it is approximately the sum of money which is required for immediate closing of all the positions. Maintenance margin is an estimation of the maximal losses of the portfolio during the next trading session. Initial margin equals maintenance margin multiplied by some coefficient which is more than or equaled to unity. To open a new short position a market participant has to have enough money on the account to cover the sum of the liquidation value and the initial margin. After clearing it is only required that enough money is left to cover the liquidation value and the maintenance margin.

The maintenance margin evaluation for option portfolio is a non-trivial problem. In 1988 Chicago Mercantile Exchange (CME) developed the methodology SPAN (Standard Portfolio Analysis of Risk) for maintenance margin calculation [19]. It has become one of the most widely used exchange margin systems in the world. The SPAN algorithm includes several calculation steps. First of them calculates the so-called Scanning risk of the portfolio. It is a rough estimation of a maximal portfolio loss during one trade session. Other steps of SPAN make the risk estimation more accurate.

To obtain the Scanning risk 16 scenarios of the concurrent changing underlying price and volatility are considered. An exchange determines a standard underlying price changing interval and implied volatility changing interval which covers all possible motions during a trading session with quite high probability for each underlying asset. The scenarios for the Scanning risk calculation are presented in Table 2 . Under scenarios $1-14$ the underlying price and volatility are changed within these standard intervals per the scenario description. CME considers the underlying price motion for 3 standard intervals for the 15th and 16th scenarios. However, only a part of the corresponding losses is considered (usually, 33\%).

An exchange clearing organization calculates losses which occur under each scenario for all traded futures and options and stores them to the so-called SPAN Risk parameter files uploaded to the relevant Internet site. Using this data, losses of all a portfolio positions are calculated and summarized for each Scanning risk scenario separately. The highest of the obtained 16 figures is the Scanning Risk of the portfolio.

In Table 3 an example of Scanning risk calculation is presented. The portfolio includes American options on Euro which are traded at CME. The underlying asset is futures on European currency. The options are quoted in dollars. There is one short Call option expiring on the 11th of February, 2011 with strike price 
Table 2. Scanning risk scenarios.

\begin{tabular}{cc}
\hline No. of Scenario & Scenario \\
\hline 1 & Underlying unchanged, volatility up \\
2 & Underlying unchanged, volatility down \\
3 & Underlying up $1 / 3$, volatility up \\
4 & Underlying up $1 / 3$, volatility down \\
5 & Underlying down $1 / 3$, volatility up \\
6 & Underlying down $1 / 3$, volatility down \\
7 & Underlying up $2 / 3$, volatility up \\
9 & Underlying up $2 / 3$, volatility down \\
10 & Underlying down $2 / 3$, volatility up \\
11 & Underlying down $2 / 3$, volatility down \\
12 & Underlying up 3/3, volatility up \\
13 & Underlying up 3/3, volatility down \\
14 & Underlying down 3/3, volatility up \\
15 & Underlying down 3/3, volatility down \\
16 & Underlying up extremely, cover part of loss \\
\hline & Underlying down extremely, cover part of loss \\
\hline
\end{tabular}

Table 3. Scanning risk example on 11.01.2011 with EUR/USD American style options.

\begin{tabular}{cccc}
\hline $\begin{array}{c}\text { No. of } \\
\text { Scenario }\end{array}$ & $\begin{array}{c}\text { Call option expiring on } \\
11 / \text { Feb/2011, } \\
\text { Strike 1.300, Losses, } \$\end{array}$ & $\begin{array}{c}\text { Put option expiring } \\
\text { on 11/Mar/2011, } \\
\text { Strike 1.200, Losses, } \$\end{array}$ & $\begin{array}{c}\text { Options portfolio } \\
\text { Losses, } \$\end{array}$ \\
\hline 1 & -605 & -428 & $-(-605)+2(-428)=-251$ \\
2 & 690 & 274 & $-(690)+2(274)=-142$ \\
3 & -1154 & -299 & $-(-1154)+2(-299)=556$ \\
4 & 139 & 300 & $-(139)+2(300)=461$ \\
5 & -123 & -578 & $-(-123)+2(-578)=-1033$ \\
6 & 1112 & 238 & $-(1112)+2(238)=-636$ \\
7 & -1769 & -188 & $-(-1769)+2(-188)=1393$ \\
8 & -539 & 317 & $-(-539)+2(317)=1173$ \\
9 & 292 & -751 & $-(292)+2(-751)=-1794$ \\
10 & 1414 & 186 & $-(1414)+2(186)=-1042$ \\
11 & -2449 & -95 & $-(-2449)+2(-95)=2259$ \\
12 & -1330 & 329 & $-(-1330)+2(329)=1988$ \\
13 & 643 & -950 & $-(643)+2(-950)=-2543$ \\
14 & 1614 & 114 & $-(1614)+2(114)=-1386$ \\
15 & -2439 & 107 & $-(-2439)+2(107)=2653$ \\
16 & 604 & -641 & $-(604)+2(-641)=-1886$ \\
\hline & & & \\
\hline & & & \\
\hline
\end{tabular}


1.300. There are also two long Put options which expire on March, 11th of the same year. The strike price of the Put options is 1.200.

The losses of options in American dollars for each scenario has been obtained from SPAN Risk parameter file for the 11th of January, 2011. The SPAN represents losses in positive figures and gains in negative figures. The fourth column of Table 3 shows the calculation of the Scanning risk of the portfolio. Scenario 15 gives the maximal loss; the Scanning risk is equal to $\$ 2653$. Both the considered option positions are out of the money. In compliance with the Risk parameter file the settlement price of the Call and Put options are \$1,912.5 and $\$ 362.5$ respectively. Therefore, the liquidation value of the portfolio on closing trading on 11.01 .2011 can be calculated as $1912.5-2 \times 362.5=\$ 1187.5$. So, the net margin (collateral) of the portfolio is $1187.5+2653=\$ 3840.5$. The account balance of an investor must be not less this sum of money for holding the positions. In opposite case margin call will be applied to the investor.

Losses of European options for Risk parameter files are calculated by exchanges using the Black-Scholes formula. For American options the model of Cox-Ross-Rubinstein [20] is commonly applied. At the same time, the procedure of the Scanning Risk calculation considered for American options is absolutely the same as that for European kind of options. The presented SPAN scenarios must be not mixed up with the scenarios for a stochastic program. Further we will consider a market where initial and maintenance margin coincide and are calculated as a Scanning risk of a portfolio. The stochastic program of option portfolio optimization formulated below includes the constraints which ensure the collateral requirements.

\section{Heuristic Approximation for the Safety-First Optimization Problem}

Chance constrained problems include constraints which have to be satisfied with some predefined probability. Many of the initial works on optimization with probability constraints belong to A. Prekopa. Papers [8], [9], [10], [11] and [13] were devoted to the problems with continuous probability distributions. In [12] a case of discrete distributions was considered.

Though, in the general case probabilistic constrained programs are not convex, in [7] a convex approximation for a broad class of continuous probabilistic constrained problems has been obtained. Exact solving methods for the problems with discrete probability distributions were in particular developed in [4], [6] and [14]. Since probabilistic constrained optimization remains a hard problem to solve, many heuristic approaches have been suggested (see, for example, [15] and [16]). Authors of [5] developed an approximate method based on random sampling of the probabilistic constraints.

In [10] the close problem of probability optimization under constraints was considered. Conformably to portfolio construction minimization of the probability of getting a return lower than some predefined level is called the safety-first optimization [21]. The solution received using the safety-first criterion 
does not take into account some scenarios, what is typical for the chance constrained programing. As the famous work [22], the paper of A. D. Roy was published in 1952 and presented the alternative approach to the portfolio optimization. It is more appropriate for option portfolio because the variance criterion is not suitable for non-linear financial instruments.

Several modern works devoted to the safety-first criterion are known. In [23] a dynamic version of the safety-first program is considered. Paper [24] ascertains that under some conditions safety-first optimization with a random target outperforms the deterministic version. In [25] the boundary estimation of Roy for solving the safety-first problem was elaborated. The case of the problem with the discrete scenarios was considered also. The program was reduced to the linear mix integer problem with binary variables.

The safety-first two-stage option portfolio optimization problem in the case of a discrete probability distribution has a generic formulation

$$
\begin{gathered}
\min _{\boldsymbol{x}, \boldsymbol{d}} \boldsymbol{p d} \\
d_{w} \in\{0,1\}, w \in \mathcal{W} \\
f_{w}(\boldsymbol{x})>Q \forall w: d_{w}=0 \\
\boldsymbol{v}^{\prime} \boldsymbol{x} \leq A
\end{gathered}
$$

Here $\boldsymbol{x}$ is a vector in $\mathfrak{R}^{1}$; its component $x_{i}$ means the recommended quantity of the financial instrument $i \in\{1, \cdots, I\}$ in the portfolio. Usual assumptions of unlimited divisibility of contracts is adopted, so, components of $\boldsymbol{x}$ are real values. Variables $d_{w}, w \in \mathcal{W}$ constitute vector $\boldsymbol{d}$. Variable $d_{w}$ is equal to unity if scenario $w$ is not taken into account (not active). Vector $\boldsymbol{p}$ contains the scenario probabilities which are summed to unity. Symbol $Q$ denotes the minimum required portfolio value at the end of the planning horizon. $f_{w}(\boldsymbol{x})$ is the value of the portfolio in the case of making the decision $\boldsymbol{x}$ under realization of scenario $w$. Finally, $\boldsymbol{v}$ is the vector of initial values of the financial instruments and $A$-available amount of money.

If $Q$ is quite large, the solution to problem (4.1), (4.2), (4.3) and (4.4) will imply inactivity of some scenarios. This is the linear mix integer program with binary variables [25]. So, only a relatively low dimension problems can be solved in an acceptable period of time. Dynamic option portfolio optimization is quite a large problem due to the considerable amount of the trading option strikes, expirations, scenarios and stages of the scenario tree. The problem with 8 strikes and one option expiration took about 5 minutes including database operations on a $2.20 \mathrm{GHz}$ computer using CPLEX 12.2 program. The entire simulation of 100 one month experiments on the management of option portfolio with 13 strikes took 98.5 hours using the mentioned facilities [17].

From this reason for optimizing the portfolio of options with several different expirations the following heuristic was suggested. We introduce auxiliary real variables $g_{w} \geq 0, w \in \mathcal{W}$, which forms vector $\boldsymbol{g}$. The safety-first mix integer problem with binary variables (4.1), (4.2), (4.3) and (4.4) can be changed by the 
following continuous linear one:

$$
\begin{gathered}
\min _{x, g} \sum_{w \in \mathcal{W}} h_{w}(\boldsymbol{p}) g_{w} \\
g_{w} \geq 0, w \in \mathcal{W} \\
f_{w}(x)+g_{w} \geq Q, w \in \mathcal{W} \\
\boldsymbol{v}^{\prime} \boldsymbol{x} \leq A
\end{gathered}
$$

Scenario $w$ is active if $g_{w}=0$ in the solution of the problem (4.5), (4.6), (4.7) and (4.8). As it will be experimentally shown, the solutions of option portfolio optimization program in form (4.1), (4.2), (4.3), (4.4) and in approximated form (4.5), (4.6), (4.7) and (4.8) can be made quite close to each other for reaching the practical purposes by the according choice of functions $h_{w}(\boldsymbol{p}), w \in \mathcal{W}$.

\section{The Problem of Option Portfolio Optimization}

Among the works on option portfolio optimization based on multistage stochastic programming the most noted is paper [26]. The authors consider an example of a portfolio optimization which includes stocks, bonds and Call options. The optimization criterion is an expected utility. Monte-Carlo simulation is used for the scenario tree building. The tree has 3 stages and 10000 scenarios. The stochastic programming results are compared with the results of static optimization with criterion of mean and variance. The superiority of the dynamic optimization over static approach to the portfolio management is indicated.

In [17] the dynamic portfolio management problem of options with one expiration date was developed. Here the problem is generalized for the portfolio of options with several expiration dates. To construct this bigger program the vector notation is used.

The stochastic program for option portfolio optimization is based on the following assumptions. Only Call and Put options of different strikes and expirations are traded; the considered market does not permit the trading of the underlying asset. A long or a short position can be opened in any option. The buying and selling prices of an option are the same. A commission is collected for each trade. The maintenance and initial margin coincide and are calculated as the Scanning Risk of the portfolio per Section 3.

\subsection{Scenario Tree}

We follow the formulation presented in particular, in [27]. Multistage stochastic program is a planning problem with horizon $\mathcal{T}$ which is a discrete set of decision stages. The beginning of the decision horizon coincides with the current time $t=0$, while $T$ denotes the end of the planning horizon. In the program of option portfolio optimization $T$ is the last expiration date of options which are considered for buying or selling. Set $\mathcal{T}$ also includes the stages which are relevant to the expirations of other option series and some stages which correspond to several intermediate dates.

Dynamics of the underlying asset price is presented by a discrete tree process 
with non-recombining sample paths. The paths branch out in the nodes of the tree. Every path concludes with a node too. Nodes along the tree, for $t \in \mathcal{T}$, are denoted by $n \in \mathcal{N}_{t}$ and for $t=0$ there is a root node which is labelled $n=0$. For $t>0$ every node $n \in \mathcal{N}_{t}$ has a unique ancestor $n$ - and for $t<T$ a non-empty set of successors $n+$. A scenario is a path from the root to a leaf node. Let $\boldsymbol{p}$ be the vector which consists of probabilities of the scenarios (or, what is the same, the leaf nodes). So, the equality $\boldsymbol{p}^{\prime} \boldsymbol{e}=1$, where $\boldsymbol{e}$ is the unit vector must be fulfilled.

\subsection{Main Notations}

\subsubsection{Decision Variables}

In the program of portfolio optimization decisions on buying and selling options can be made in the nodes of the scenario tree. Let $\boldsymbol{x}_{n}^{+} \geq 0$ denotes the column vector of variables which determine the quantities of buying options in node $n$ of the tree. The similar vector for selling options is denoted by $\boldsymbol{x}_{n}^{-} \geq 0$. We follow the assumption about unbounded divisibility of option contracts, so, the components of vectors $\boldsymbol{x}_{n}^{+}$and $\boldsymbol{x}_{n}^{-}$are real numbers. Vectors $\boldsymbol{x}_{n}^{+}$and $\boldsymbol{x}_{n}^{-}$have the same structure: at first the elements follow which accords with Call and Put options expiring in the current month, then in the next month and so on. As the options are not traded on the expiration date, if stage $t$ corresponds to such a date, the relevant elements of vectors $\boldsymbol{x}_{n}^{+}$and $\boldsymbol{x}_{n}^{-}$are set to zero. So, for $t=T$ vectors $\boldsymbol{x}_{n}^{+}$and $\boldsymbol{x}_{n}^{-}$are zero vectors always.

\subsubsection{Model Variables}

Column vectors $\boldsymbol{x}_{n}$ contain the sizes of open option positions in the portfolio in node $n$ of the tree after the adjustment of the portfolio. Vectors $x_{n}$ are organized similarly to $\boldsymbol{x}_{n}^{+}$and $\boldsymbol{x}_{n}^{-}$. Negative elements of $\boldsymbol{x}_{n}$ corresponds to short option positions.

Besides, the portfolio contains a cash account. The account balance in node $n$ after the portfolio adjustment will be denoted by $A_{n}$. The liquidation value of the options in node $n$ of the tree after the adjustment of the portfolio is denoted by $L_{n}$. We will also use notation $C_{n}$ for the corresponding portfolio adjustment cost. For formulation of optimization criterion, we will need portfolio value at the end of the planning horizon depending on the scenario which will be denoted by $V_{n}, n \in \mathcal{N}_{t}$.

Variables $M_{n}$ will present the initial margin of the portfolio in the corresponding nodes of the tree. Per the heuristics suggested in Section 3 we introduce vector $\boldsymbol{g}$ of auxiliary variables $g_{n} \geq 0, n \in \mathcal{N}_{t}$, associated with the relevant scenarios.

\subsubsection{Random Data}

Let $v_{n}$ be the column vector of the options prices in node $n$ of the tree. It has the same structure as vectors $\boldsymbol{x}_{n}^{+}, \boldsymbol{x}_{n}^{-}$and $\boldsymbol{x}_{n}$. Because components of vector $v_{n}$ depend on the scenario of the underlying asset price, they are random data. 


\subsubsection{Program Constants}

Symbol $c$ denotes a commission which is levied for each trade. So, though there is no spread between the buying and the selling prices, it is necessary to pay more than the true price to buy an option and the amount less than the true price will be received after selling the option. It models transaction costs at our market. The commission is not paid when options expire.

Let $\lambda_{n}$ be the matrix of options price losses which refer to different SPAN scenarios in node $n$. Each matrix $\lambda_{n}$ includes 16 rows in accordance with the scenarios of Table 2. First columns of the matrix correspond to the options expired in the nearest month, after these the columns follow which are relevant to the next month's expiration options.

The real cash balance of the portfolio before its adjustment at the current moment which is presented by the root node of the tree will be denoted by $A_{0-}$. Similarly, vector $\boldsymbol{x}_{0-}$ presents current option positions by the moment of the portfolio optimization. As denoted above, $Q$ is the minimum required portfolio value at the end of the planning horizon.

\subsection{Constraints and Object Functions}

The stochastic program of option portfolio optimization includes the following constraints.

\subsubsection{Constraints of Non-Negativity of Variables}

As already mentioned, the decision variables and the auxiliary variables must not be less than zero:

$$
\begin{gathered}
\boldsymbol{x}_{n}^{+} \geq 0, \boldsymbol{x}_{n}^{-} \geq 0, n \in \mathcal{N}_{t}, t \in \mathcal{T} \\
g_{n} \geq 0, n \in \mathcal{N}_{T}
\end{gathered}
$$

It is also necessary to ensure that the account balance cannot be negative in any situation, so

$$
A_{n} \geq 0, n \in \mathcal{N}_{t}, t \in \mathcal{T}
$$

\subsubsection{Problem Equations}

The quantity of options in the portfolio in node $n$ after the portfolio adjustment is given by the following recursions:

$$
\boldsymbol{x}_{n}=\boldsymbol{x}_{n-}+\boldsymbol{x}_{n}^{+}-\boldsymbol{x}_{n}^{-}, n \in \mathcal{N}_{t}, t \in \mathcal{T}
$$

For the root node of the tree we have $\boldsymbol{x}_{0}=\boldsymbol{x}_{0-}+\boldsymbol{x}_{0}^{+}-\boldsymbol{x}_{0}^{-}$. Here $\boldsymbol{x}_{0-}$ is the vector introduced in section 5.2.4 which contains sizes of factual option position before the portfolio optimization.

The portfolio adjustment costs are given by

$$
C_{n}=\boldsymbol{v}_{n}^{\prime}\left(\boldsymbol{x}_{n}^{+}-\boldsymbol{x}_{n}^{-}\right)+c \boldsymbol{e}^{\prime}\left(\boldsymbol{x}_{n}^{+}-\boldsymbol{x}_{n}^{-}\right), n \in \mathcal{N}_{t}, t \in \mathcal{T}
$$

Here $\boldsymbol{e}$ is a unit vector the dimension of which coincides with the dimension of vectors $\boldsymbol{x}_{n}^{+}$and $\boldsymbol{x}_{n}^{-}$.

The portfolio liquidation values are given by the equations 


$$
L_{n}=-\boldsymbol{v}_{n}^{\prime} \boldsymbol{x}_{n}, n \in \mathcal{N}_{t}, t \in \mathcal{T}
$$

After the portfolio adjustment in node $n$ the account balance will be

$$
A_{n}=A_{n-}-C_{n}, n \in \mathcal{N}_{t}, t \in \mathcal{T}
$$

For the root node of the tree the equation takes the form $A_{0}=A_{0-}-C_{0}$, where $A_{0-}$ is the factual cash balance before portfolio optimization (see Section 5.2.4).

Further we will need values of the portfolio at the final stage $T$ :

$$
V_{n}=A_{n}-L_{n}, n \in \mathcal{N}_{T}
$$

\subsubsection{Budget Constraints}

The initial margin for node $n$ is given by variable $M_{n}$ which must satisfy the following 16 inequalities:

$$
M_{n} \geq \lambda_{n} x_{n}
$$

Inequalities (5.9) mean that variable $M_{n}$ is not less than any element of the column vector in the right hand side which is the portfolio loss referring to the corresponding SPAN scenario. If $M_{n}$ is equal to one of the elements, so $M_{n}$ equals the Scanning risk of the portfolio [28]. Inequalities (5.9) are introduced for all $n \in \mathcal{N}_{t}, t \in \mathcal{T}$.

To prevent margin call, the account balance after the portfolio adjustment must not be less than the required collateral. So, the problem includes the budget constraints:

$$
A_{n} \geq L_{n}+b M_{n}, n \in \mathcal{N}_{t}, t \in \mathcal{T}
$$

where $b>0$ is a reserve coefficient which increases the initial margin value. It prevents margin call during option portfolio management due to inaccuracy of the optimization program.

We consider two versions of the object function of the problem. First is an exact criterion which is relevant to the generic problem (4.1)-(4.4). We have

$$
\begin{aligned}
& \text { 5.3.4. Exact Chance Constraints and Safety-First Object Function } \\
& \qquad \begin{array}{c}
V_{n} \geq Q \forall n \in \mathcal{N}_{T}: d_{n}=0 \\
\min _{\boldsymbol{x}, \boldsymbol{d}} \boldsymbol{p}^{\prime} \boldsymbol{d}
\end{array}
\end{aligned}
$$

The approximation version of the safety-first criterion accords with the problem (4.5)-(4.8).

\subsubsection{Approximative Chance Constraints and Safety-First Object Function}

Following (4.8), we introduce the constraints

$$
V_{n}+g_{n} \geq Q, n \in \mathcal{N}_{T}
$$

Scenario associated with leaf node $n \in \mathcal{N}_{T}$ is active if $g_{n}=0$.

Per (4.5), the approximated safety-first criterion is the weighted value of variables $g_{n}, n \in \mathcal{N}_{T}$ :

$$
\min _{\boldsymbol{x}, \boldsymbol{g}} \sum_{n \in \mathcal{N}_{T}} h_{n}(\boldsymbol{p}) g_{n}
$$




\section{Case Study: Portfolio Management Using the Stochastic Program}

\subsection{Case Study Assumptions}

We adopt the following additional assumptions about the option market for the case study. The underlying asset does not pay any dividend. The trading goes on every day; there are no days of rest or holidays. On-line trading is not considered here: each day is presented as a point in time. Each month consists of 21 days, so, a year includes 252 trading days. The options expire on the 21 st day of the relevant expiration month. Options are not traded on their expiration date.

The option underlying asset price follows a geometric Brownian motion. The volatility of the underlying asset is constant. The drift equals the risk-free interest rate, so it is a risk-neutral world. The risk-free rate is the same for any horizon, so, the interest rate curve is flat.

The simulation considers some liquidity features of real option markets also. If the underlying price is changed, an exchange can introduce additional options into trading so that the sufficient quantity of the strikes above and below the current underlying price are always accessible for trading. On the other hand, the liquidity of deep out of the money options and deep in the money options can collapse. In this connection at any day we permit trading of only such options for which the strikes belong to the interval

$$
S \mathrm{e}^{\left(r-\sigma^{2} / 2\right) t \pm 3 \sigma \sqrt{\tau}}
$$

where $r$ is a risk-free rate, $\tau$ is time to their expiry.

The options are quoted per the Black-Scholes model. Values of the elements of matrixes $\lambda_{n}$ are calculated on the basis of the Black-Scholes formula too using SPAN scenarios from Table 2.

As the underlying price follows the geometric Brownian motion, the scenario tree at every stage has to approximate the corresponding log-normal probability distribution. Successors $n+$ of each node (besides terminal stage nodes) exhibit underlying prices equaled to the option strike prices which belong to interval (5.15). Some intermediate asset prices are also presented as well as two price values beyond interval (5.15) for the approximation of the distribution tails. All exhibited prices are equidistant.

The tree includes stages which correspond with the option expiration dates and with some intermediate dates. The detailed description of the scenario tree construction was given in [17].

In our experiments the scenario tree will have from 2 to 4 stages and different number of the scenarios depending on the simulated day of month, number of expirations of options in the portfolio and the current underlying price. We will indicate the corresponding structures of the trees for the case studies in Sections 6.2 and 6.3 .

First of all, we discuss choice of functions $h_{n}(\boldsymbol{p})$. Then the strategy of using the program (5.1)-(5.10), (5.13)-(5.14) for option portfolio management are 
suggested. Initial data, structures of the scenario trees and results of Monte-Carlo simulation of option portfolio management are provided.

\subsection{Choice of Weight Functions for the Approximated Safety-First Criterion}

Different power functions $h_{n}(\boldsymbol{p})$ for objective function (5.14) have been tested. As the experiments have shown, the most accurate results are obtained using the simple weights equaled to $p_{n}^{2}, n \in \mathcal{N}_{T}$. To illustrate these weights, in Figure 2 the values of variables $g_{n}, n \in \mathcal{N}_{T}$ are shown for one considered example. Optimization problem (5.1)-(5.10), (5.13)-(5.14) has been solved. The according scenario tree had 3 stages and 147 scenarios. The portfolio included options with one expiration date as in Golembiovsky \& Abramov 2013. In spite of the fact that many of the variables $g_{n}$ are different from zero, the summarized probability of inactive scenarios was 0.0178 during solving the approximate problem with $p_{n}^{2}$ as the weights in the optimization criterion. The correspondent exact mix integer optimization problem with binary variables and chance constraints (5.11) and criterion (5.12) provided the probability value 0.0140 .

To compare results of option portfolio management based on the exact optimization problem (5.1)-(5.12) and on the approximated problem (5.1)-(10), (5.13)-(5.14) we also simulated management of the portfolio which included options of one expiration date. In each experiment one year of portfolio management was simulated. When options expired in a certain month, the portfolio started from the residual account balance. We simulated 10 1-year tracks for both approaches. The parameters of the market and of the program are given in Table 4. So, the risk-free rate was set to zero. 30 option strikes were considered but as it was mentioned only those were traded at any given moment which belong to interval (5.15). Minimal required portfolio value $Q$ for constraints (5.11) and (5.13) was calculated based on the data of Table 4 as $A_{0-} \times y \times \tau$, where $\tau$ is the time from the beginning of the year till the end of the planning horizon.

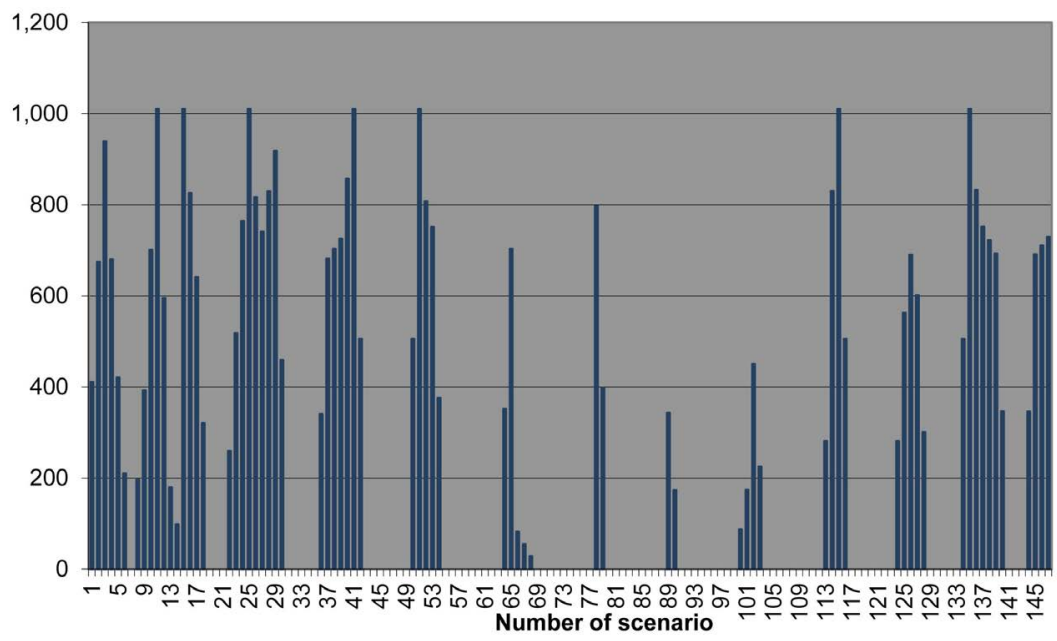

Figure 2. Values of variables $g_{n}, n \in \mathcal{N}_{T}$ in the result of one expiration option portfolio optimization. 
Table 4. Parameters of simulation of option portfolio management.

\begin{tabular}{cc}
\hline Parameter & Value \\
\hline Volatility of the underlying asset price, $\sigma$ & 0.1 \\
Risk-free rate, $r$ & 0.0 \\
Lower and upper limits of traded option strikes & $\$ 72 ; \$ 130$ \\
Differences between strikes & $\$ 2.00$ \\
Underlying asset price on day 1 & $\$ 100.00$ \\
Initial account balance, $A_{0-}$ & $\$ 1000.00$ \\
Commission for one trade, $c$ & $\$ 0.001$ \\
Margin reserve coefficient, $b$ & 5 \\
Minimum required portfolio return per year, $y, \%$ & 6
\end{tabular}

To decrease experiment time, the portfolio was adjusted on the 7 th and on the 14th day of each month only. For the optimization on the 7th day the relevant scenario tree included three stages, which related to the current day, the 14th day and the 21st day of the current month. Under portfolio optimization on the 14th day of a month the tree had only two stages: for the current 14th day and for the 21 st day of the month.

As it can be seen in Figure 3 and Figure 4, using the exact problem led to only one failed track and using the heuristics we obtained two failed tracks. All other experiments gave the required profitability. At the same time, solving the exact problem for maximum scenario tree took about 26 seconds, while the approximate problem required 8 seconds to solve. All the experiments with the exact problem took about 19 hours, with the heuristic method-about only 8 hours. The advantage grows quickly when the dimension increases. This speed up allowed us to deal with option portfolios with more number of expirations.

\subsection{Option Portfolio Management: The Strategy and Simulation Results}

Theoretically, the stochastic program (5.1)-(5.10), (5.13)-(5.14) permits optimization of an option portfolio with any number of expirations. So, it is possible to set a planning horizon to the entire investment period, for example, one year. However, the dimension problem becomes essential with addition of expirations even with employment of the suggested heuristics. Besides, as experience shows, managing the portfolio during such a long period results in the portfolio swelling up: it accumulates too many positions and loses controllability. To prevent the swelling up effect, traders of real markets make efforts to cash as often as possible by waiting for option expiration. To get cash in such a way as often as possible using only options of one nearest expiration is expedient. However, this approach does not provide a quite low probability of loss for practical purposes [17]. Here we examine the strategy of applying the program (5.1)-(5.10), (5.13)-(5.14) which assumes including options of one or two expirations in the portfolio. 


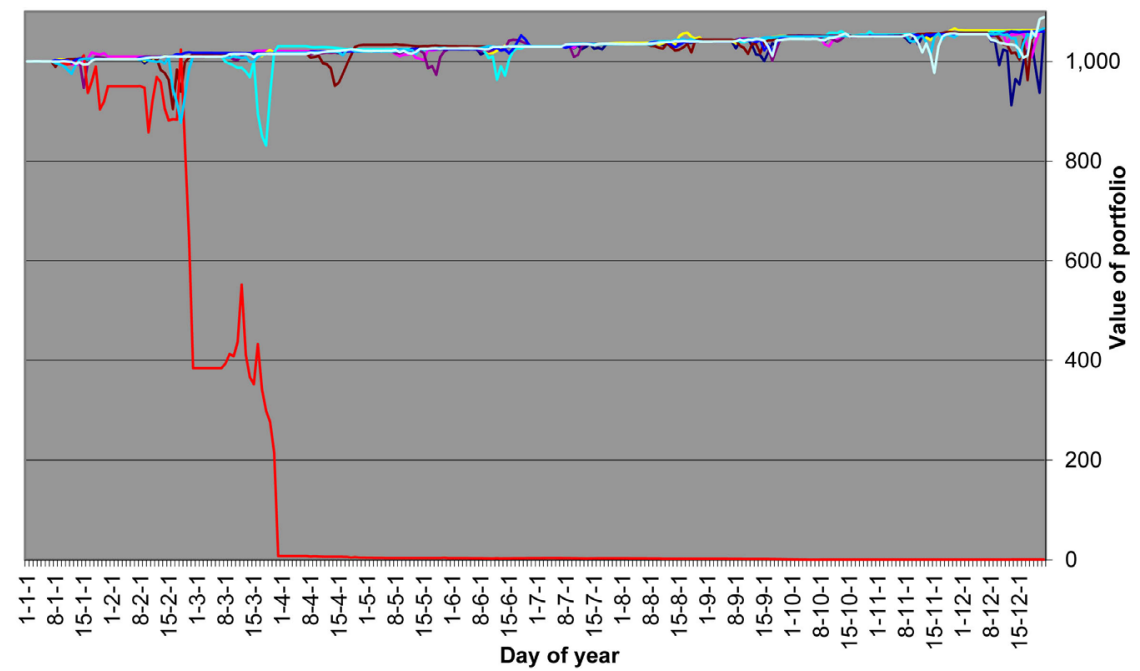

Figure 3. Results of portfolio management simulation. One expiration option portfolio. Exact optimization mix integer problem with binary variables has been solved.

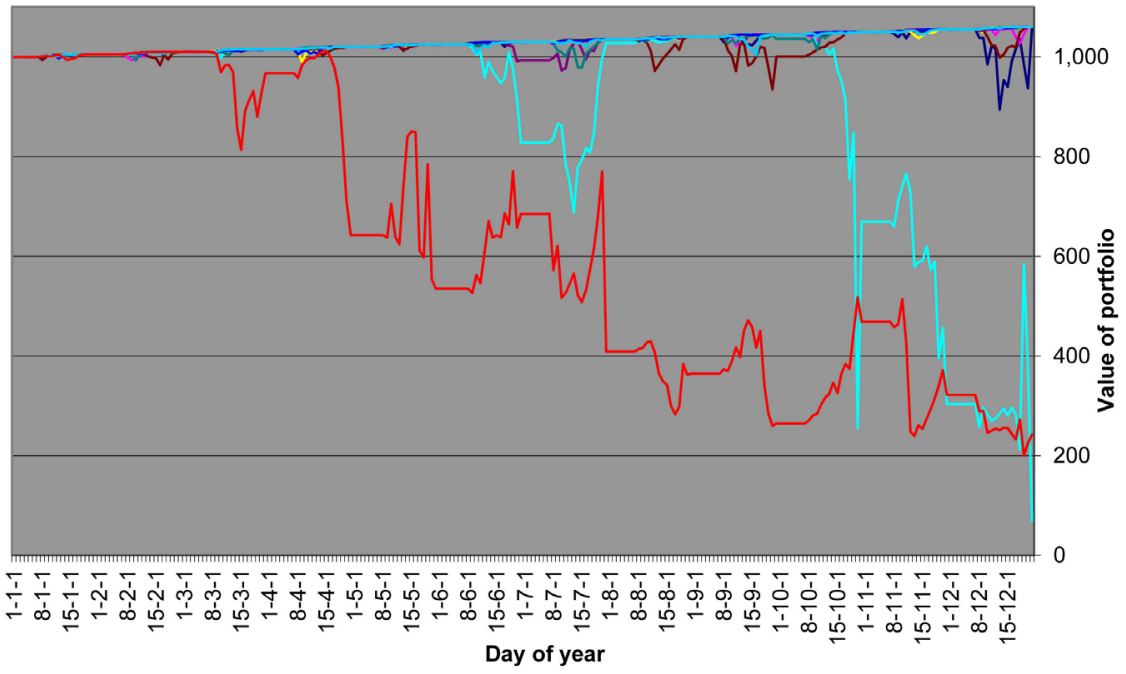

Figure 4. Results of portfolio management simulation. One expiration option portfolio. Approximate linear optimization problem has been solved.

The same values of the simulation parameters from Table 4 were used. The following strategy of the portfolio management was implemented. The portfolio was created on the first day of the first month. The portfolio was optimized and adjusted every second day (all odd dates). Until the 7th day only options of one expiration were included in the portfolio. Starting from the 7th day, if the probability of not reaching the required portfolio value was more then 0.001 , the options of the next expiration were included for optimization. Under this conditions the planning horizon was shifted to the end of the next month.

If the options of the second expiration were not opted for, the portfolio expired at the end of the current month. The following simulation started from the obtained sum of money. In the opposite case the portfolio continued to the next month. Then the same algorithm of the portfolio management was repeated: if 
starting from the 7th day of the new month the probability of providing the required portfolio value was not proved to be satisfactory, then the options which expire in the next month were added to the portfolio.

The scenario trees which were constructed for the described optimizations are presented in Table 5. During the portfolio optimization on the 1st, 3rd and 5th day of any month the scenario tree had the structure $t-7-14-21$. Here $t$ means that the root node of the tree corresponding to the current day; "7", "14" and " 21 " mean that the second, third and fourth stages of the tree associate with the 7 th, 14th and 21 st day of the current month.

Starting from the 7th day, if the options of the next expiration were not included in the optimization problem, the tree could have the structures $t-14-21$ or $t$-21. In the case of adding the next expiration options to the portfolio, the planning horizon was shifted to the end of the next month. The according tree structure can be presented as $t-14-21-21$ or $t-21-21$, i.e. the last stage of the tree accords with the 21 st day of the next month. If we optimize on the 21 st day, the planning horizon is the end of the next month and the according tree has the structure $t-7-14-21$.

The described strategy was used for each simulated year. At the end of each year the portfolio was closed. Overall we simulated 101 -year tracks of the portfolio management. It took about 71.5 hours on the fore mentioned computer. The results are illustrated in Figure 5. The notation "1- $x$ " means the 1st day of month " $\mathrm{x}$ " of a year. As it can be seen, the volatility of the portfolio is quite high and some tracks developed an essential drawdown. However, all tracks had a positive trend and contrary to [17] none of them finished with a loss. It can be concluded that the resulting probability of success of the portfolio management is quite close to unity.

Let's consider an example of the option portfolio adjustment. The situation occurred on the 7th day of the first month. The according track is shown in

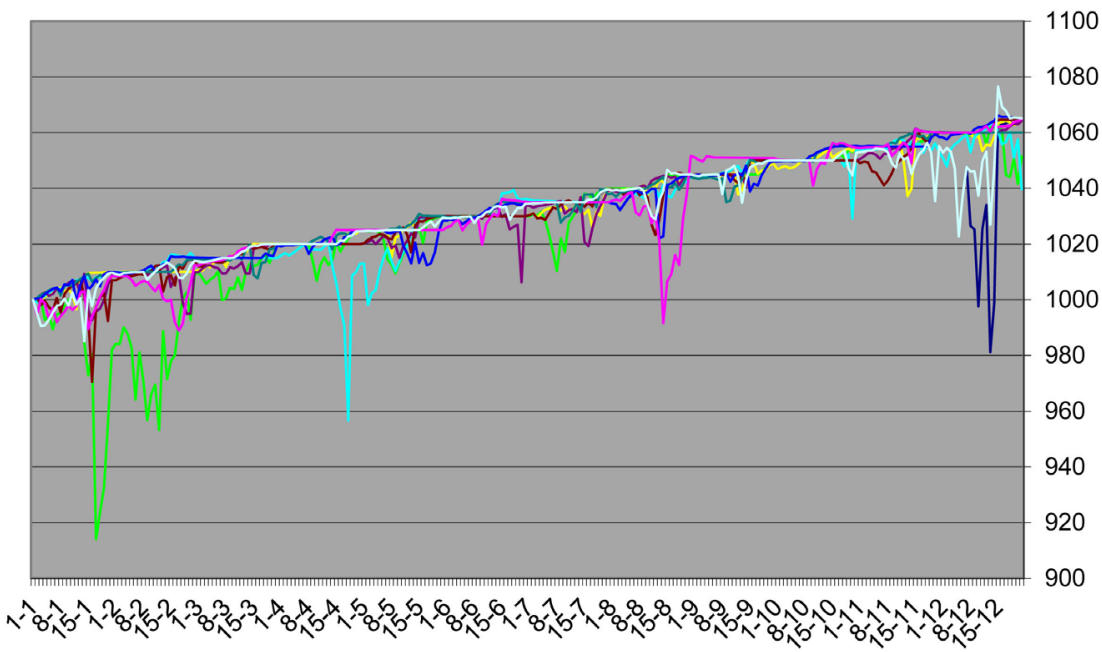

Figure 5. Results of option portfolio management simulation. One or two expiration dates. Approximate linear optimization problem was sold. 
Table 5. Scenario tree structures.

\begin{tabular}{ccc}
\hline Day of the current month & \multicolumn{2}{c}{ Scenario tree structure } \\
\hline Horizon & End of the current month & End of the next month \\
\hline 1,3 or 5 & $t-7-14-21$ & $t-14-21-21$ \\
$7,9,11$ or 13 & $t-14-21$ & $t-21-21$ \\
$15,17,19$ & $t-21$ & $t-7-14-21$ \\
21 & & \\
\hline
\end{tabular}

green in Figure 5. The scenario tree used under the first optimization included 3 stages and 149 scenarios. The obtained probability of not reaching the required return was 0.00402 , which is more than the adopted level 0.001. In accordance with the described strategy the portfolio optimization including options of the next expiration was also realized. The planning horizon was set to the end of the second month. The relevant scenario tree had 4 stages and 2964 scenarios. The second optimization provided the probability of not reaching the required return of 0.00274 . It is more than the required level 0.001 , nevertheless, according to the strategy, the recommendations were realized under the simulation and the portfolio was prolonged to the next month.

Table 6 contains option portfolio positions on the 7th day and recommendations on the portfolio adjustment received by optimization of one expiration month portfolio and the portfolio with the options of two expiration dates. Actually, only one option of the next expiration was recommended for inclusion in the portfolio. It can be seen that the portfolio included short and long positions in Call and Put options not far from the money (underlying price on this day was $\$ 98.51$ ). Figure 6 shows the relation between the portfolio value before an adjustment and taking into account the recommendations received during the first optimization and the second optimization on the 7th day of the first month. It is evident that short positions prevailed in the portfolio in all cases. We can conclude that the portfolios created by the stochastic program include mostly short positions and, consequently, they are theta-positive [18].

Figure 7 illustrates the discussed track till the end of the year. It shows the graph of the portfolio value again and the values of the probability of failure in the first and the second optimization which are shown in blue and in red accordingly. The values of the probabilities are shown on the left vertical axis. If the red bar is absent, it means that the planning horizon was not resettled. We can see, that it was only once in the 6th month, the planning horizon was resettled 11 times during the simulated year. It can also be noted that not always the probability of failure after the horizon resettling is less than in the optimization the portfolio with options of one expiration. So, the roll-over of the planning horizon does not necessarily increase the probability providing the required return, it rather prolongs the process of the management what gives a chance to improve the situation. 


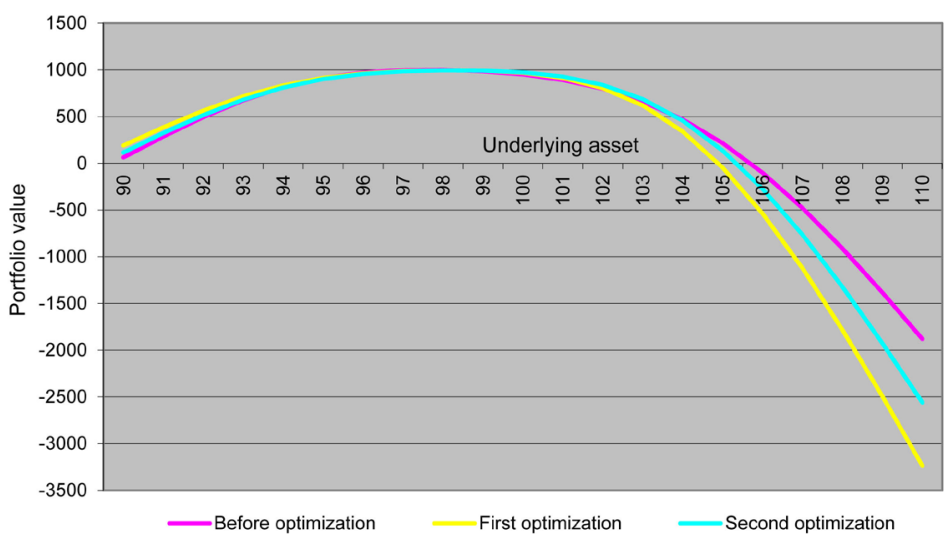

Figure 6. Values of the portfolio depending on the underlying price.

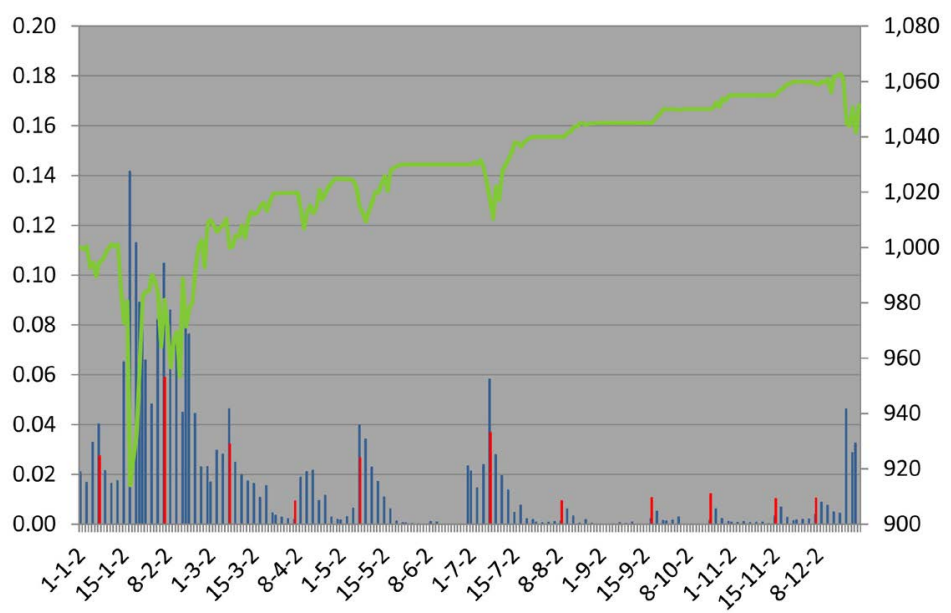

Figure 7. One simulated track of option portfolio management.

Table 6. Portfolio and results of optimization.

\begin{tabular}{|c|c|c|c|c|c|c|c|c|}
\hline \multirow{3}{*}{ Strike, \$ } & \multirow{3}{*}{ Option } & \multirow{3}{*}{ Position } & \multirow{2}{*}{\multicolumn{2}{|c|}{ One expiration }} & \multicolumn{4}{|c|}{ Two expirations } \\
\hline & & & & & \multicolumn{2}{|c|}{ First month options } & \multicolumn{2}{|c|}{ Second month options } \\
\hline & & & Buy & Sell & Buy & Sell & Buy & Sell \\
\hline 92 & Call & -5.07 & & & & & & \\
\hline 92 & Put & & & & & & & \\
\hline 94 & Call & -206.67 & & & & 274.73 & & \\
\hline 94 & Put & & & & & & & \\
\hline 96 & Call & -44.86 & 33.36 & & & & & \\
\hline 96 & Put & & 10.44 & & & & & \\
\hline 98 & Call & & & 7.98 & & & 10.76 & \\
\hline 98 & Put & & 3.98 & & & & & \\
\hline 100 & Call & -3.74 & & & & & & \\
\hline 100 & Put & 28.89 & 15.27 & & & & & \\
\hline 102 & Call & -187.39 & & & & 61.72 & & \\
\hline 102 & Put & & & & & & & \\
\hline 104 & Call & & & 284.00 & & 229.76 & & \\
\hline 104 & Put & -11.22 & & & & 264.19 & & \\
\hline 106 & Call & -77.77 & & & & & & \\
\hline 106 & Put & -254.82 & & & & & & \\
\hline
\end{tabular}




\section{Practical Implications}

The statistical arbitrage based on the planning horizon roll-over is used in the real option exchange trading. The procedure looks like the example considered in section 2. Traders usually short a strangle and transfer its leg when the underlying price approaches the strike of the corresponding options. The transfer to much further strike or to the next expiration month can be fulfilled. During this, the number of options in the leg increases. The stochastic program and the strategy developed in this article permits us to obtain more complex portfolios which include short and long positions.

The analysis of this article is restricted by the assumption of constant volatility which is never observed in real markets. The results of the paper can be used as a basis for developing more efficient strategy of the statistical arbitrage with the horizon rolling-over. To develop the stochastic program for real option trading, an appropriate model of underlying price with varying volatility should be constructed. The scenario tree of the stochastic program must approach the according dynamics of an underlying price.

\section{Conclusions}

An approach for making return which exceeds the risk-free rate in a risk-neutral world via option portfolio management has been considered. A stochastic program with the safety-first criterion has been developed for portfolio optimization. It permits taking options of arbitrary number of different expiration dates in the portfolio. The program uses the following assumptions which are reasonable and close to the conditions of the real option markets:

- Only Call and Put options with different strikes and expirations are traded; a long or short position can be opened in any option,

- The buying and selling prices of an option are the same. However, a commission is collected for each trade. So, the proceeds received by selling is less than the amount of money paid when the option is bought,

- The maintenance and initial margin of a portfolio coincide and are calculated according to the first step of the methodology of Standard Portfolio Analysis of Risk which is called the Scanning risk.

The safety-first criterion ensures minimization of the probability of receiving a return below the predefined level by not considering some scenarios with low probability. It leads to a mix integer program with binary variables, which is a hard problem to solve. To optimize the portfolio with options of few expirations, a heuristic approach has been suggested. It reduces the problem to an approximate continuous linear optimization problem which requires much less time to solve.

The results of the exact and approximate problem were found to be close to each other, which were proved by computer experiments. In particular, we simulated 101 -year tracks of the single expiration option portfolio management using the exact program for the portfolio optimization and the approximate 
program. In the first case 9 out of 10 tracks gave the planed return and one track finished with a loss (Figure 3). In another experiment, there were 8 successful and two failed tracks (Figure 4). At the same time the experiment with the exact program took about 19 hours, with the heuristic method-only about 8 hours on the $2.20 \mathrm{GHz}$ computer using CPLEX 12.2 program. This speed up allowed us to do the case study of option portfolio with more than one expiration.

The following main additional assumptions were adopted for the case study realization:

- The underlying price variation accords with the geometric Brownian motion. The drift is equaled to the risk-free rate, which is the same for all the periods,

- Options are quoted on the basis of the Black-Scholes formula so, it is the risk-neutral world where the statistical arbitrage based on the difference between the objective and risk-neutral probability measure is impossible,

- The interest rate curve is flat.

The following portfolio management strategy was used. The portfolio started from the account balance by trading options of the nearest expiration. The planning horizon accorded with the expiration of the options (last day of the current month). The portfolio was optimized every two days. If starting from the 7th day of the month the probability of not reaching the predefined return level was shown as more than 0.001 , the planning horizon was shifted to the end of the next month. The portfolio was optimized taking into account options expiring in the next month and the obtained recommendations were realized. This offered the possibility of improving a falling portfolio. Then the strategy was repeated. If the options of the second expiration were not opted for, the portfolio expired at the end of the current month. The following simulation started with the obtained sum of money.

Table 6 shows an example of the portfolio and recommendations on its restructuring. It is seen that the program kneads more complex portfolios than the traditional combinations such as straddle, strangle, bull and beer spreads, butterfly spread and so on.

101 -year tracks of option portfolio management were simulated and none of them finished with a loss (Figure 5). They provided a return close to the required level (6\% per year) however the risk-free rate was zero. It does not contradict the fact that in a risk-neutral world the expected return of option portfolio accords with the risk-free rate. The reason is the following: the probability of getting the required return is so close to unity that the case of receiving a negative return has not been realized during the simulated 10 years.

The conducted research has shown that it is possible to make more than the risk-free rate in a risk-neutral world in some sense. The developed approach to the statistical arbitrage is based on the roll-over of option portfolio. The developed portfolio management strategy can be used as a basis for constructing more effective trading strategies for the real option markets. The paper can be useful for managers of option portfolios. 


\section{Conflicts of Interest}

The authors declare no conflicts of interest regarding the publication of this paper.

\section{References}

[1] Shiryaev, A.N. (1999) Essentials of Stochastic Finance: Facts, Models, Theory. Advanced Series on Statistical Sciences and Applied Probability, Vol. 3, World Scientific. https://doi.org/10.1142/3907

[2] Javaheri, A. (2005) Inside Volatility Arbitrage: The Secrets of Skewness. John Wiley and Sons Inc., New Jersey.

[3] Lindberg, P. (2010) Optimal Partial Hedging in a Discrete-Time Market as a Knapsack Problem. Mathematical Methods of Operations Research, 72, 433-451. https://doi.org/10.1007/s00186-010-0327-0

[4] Beraldi, P. and Ruszczynski, A. (2002) A Branch and Bound Method for Stochastic Integer Problems under Probabilistic Constraints. Optimization Methods and Software, 17, 359-382. https://doi.org/10.1080/1055678021000033937

[5] Campi, M.C. and Gatatti, S. (2011) A Sampling-and-Discarding Approach to ChanceConstrained Optimization: Feasibility and Optimality. Journal of Optimization Theory and Applications, 148, 257-280. https://doi.org/10.1007/s10957-010-9754-6

[6] Dentcheva, D., Prekopa, A. and Ruszczynski, A. (2000) Concavity and Efficient Points for Discrete Distributions in Stochastic Programming. Mathematical Programming, 89, 55-79. https://doi.org/10.1007/PL00011393

[7] Nemirovski, A. and Shapiro, A. (2006) Convex Approximations of Chance Constrained Programs. SIAM Journal on Optimization, 17, 969-996.

https://doi.org/10.1137/050622328

[8] Precopa, A. (1970) On Probabilistic Constrained Programming. Proceedings of the Princenton Symposium on Mathematical Programming, Princeton University Press, 113-138.

[9] Prekopa, A. (1973) Contribution to the Theory of Stochastic Programming. Mathematical Programming, 4, 202-221. https://doi.org/10.1007/BF01584661

[10] Prekopa, A. (1995) Programming under Probabilistic Constraint and Maximizing Probabilities under Constraints. Mathematics and It's Applications, 324, 319-371.

[11] Prekopa, A. (1995) Stochastic Programming. Kluwer Academic Publishers, Boston. https://doi.org/10.1007/978-94-017-3087-7

[12] Prekopa, A., Vizvari, D. and Badics, T. (1998) Programming under Probabilistic Constraint with Discrete Random Variable In: Giannessi, F., Komlosi, S. and Rapcsak, T., Eds., New Trends in Mathematical Programming, Applied Optimization, Springer, Berlin, 13, 235-255.

[13] Prekopa, A. (2003) Probabilistic Programming In: Ruszczynski, A. and Shapiro, A., Eds., Handbooks in Operations Research and Management Science, Elsevier, 10. https://doi.org/10.1016/S0927-0507(03)10005-9

[14] Ruszczynski, A. (2002) Probabilistic Programming with Discrete Distributions and Precedence Constrained Knapsack Polyhedra. Mathematical Programming, 93, 195 215. https://doi.org/10.1007/s10107-002-0337-7

[15] Tanner, M.W. and Beier, E.B. (2008) A General Heuristics Method for Joint Chance-Constrained Stochastic Programs with Discretely Distributed Parameters. http://citeseerx.ist.psu.edu/viewdoc/summary?doi=10.1.1.398.6433 
[16] Watson, J.-P., Wets, R.J.-B. and Woodruff, D.L. (2010) Scalable Heuristics for a Class of Chance-Constrained Stochastic Programs. INFORMS Journal on Computing, 4, 543-554. https://doi.org/10.1287/ijoc.1090.0372

[17] Golembiovsky, D. and Abramov, A. (2013) Option Portfolio Management as a Chance Constrained Problem. In: Gassmann, H., Wallace, S.W. and Ziemba, W.T., Eds., Stochastic Programming. Applications in Finance, Energy, Planning and Logistics, World Scientific, 155-172.

[18] Hull, J.C. (2012) Options, Futures and Other Derivatives. 8th Edition, Prentice Hall, Prentice.

[19] Chicago Mercantile Exchange (1999) The Standard Portfolio Analysis of Risk (SPAN) Performance Bond System at the Chicago Mercantile Exchange. Technical Specification.

[20] Cox, J.C., Ross, R.A. and Rubinstein, M. (1979) Option Pricing: A Simplified Approach. Journal of Financial Economics, 7, 229-263. https://doi.org/10.1016/0304-405X(79)90015-1

[21] Roy, A.D. (1952) Safety First and the Holding of Assets. Econometrica, 431-450. https://doi.org/10.2307/1907413

[22] Markowitz, H. (1952) Portfolio Selection. The Journal of Finance, 7, 77-91.

[23] Prigent, J.-L. and Toumi, S. (2005) Portfolio Management with Safety First Criteria in Complete Financial Markets. International Journal of Business, 10, 233-250.

[24] Singer, N. (2010) Safety-First Portfolio Optimization: Fixed versus Random Target. https://www.econstor.eu/bitstream/10419/74652/1/747604916.pdf

[25] Norkin, V.I. and Boyko, S.V. (2012) Safety-First Portfolio Selection, Cybern and Syst. Analysis, 48, 180-191. https://doi.org/10.1007/s10559-012-9396-9

[26] Carino, D.R. and Turner, A.L. (1998) Multiperiod Asset Allocation with Derivative Assets. In: Ziemba, W.T. and Mulvey, J.M., Eds., Worldwide Asset and Liability Modeling, Cambridge University Press, Cambridge, 129-151.

[27] Consigli, G., Iaqinta, G. and Morrigia, V. (2012) Retirement Planning in Individual Asset-Liability Management. IMA Journal of Management Mathematics, 23, 365-396. https://doi.org/10.1093/imaman/dps019

[28] Golembiovskii, D.YU. and Dolmatov, A.S. (2001) An Optimization Model for a Portfolio of Financial Derivative Instruments with Pledge Limitations. Journal of Computer and System Sciences International, 40, 425-435. 